\title{
Bald allein am Stuhl?
}

In den vergangenen Wochen ergaben sich einige entscheidende personalpolitische Veränderungen: Rückwirkend zum 01.01.2015 wurden in etlichen Kammerbereichen die Tarifverträge für zahnmedizinische Fachangestellte (ZFA) angepasst. Auch wenn dies auf den ersten Blick keine entscheidende Information für Studenten oder frisch approbierte Zahnärzte zu sein scheint, gilt es, das Thema noch einmal näher zu beleuchten.

\section{Dr. Philipp F. Gebhardt //}

BISS45 - Kieferorthopädie Berlin www.biss45.de

Dr. Gebhardt berichtet in jeder Ausgabe über seinen Weg vom abgeschlossenen Studium zum jungen Zahnarzt.

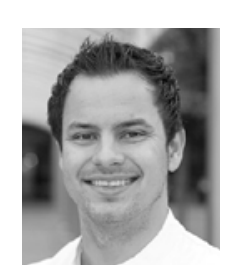

$\mathrm{E}$ in gesunkenes Interesse an dem Beruf der zahnmedizinischen Fachangestellten zeichnete sich schon in den letzten Jahren durch drastisch fallende Absolventenzahlen ab. Sicher ist diese Entwicklung multifaktoriell bedingt. Aufgrund der Fakten, welche Schwierigkeiten sich bei der Suche nach guten Mitarbeitern ergeben, liegt es jedoch auf der Hand, dass die Tarifverträge nicht mehr zeitgemäß waren und sicher einer der Hauptgründe für diesen negativen Verlauf darstellten. Eine Anpassung der Gehälter der ZFA war somit längst überfällig und wird auch weiterhin notwendig sein. Deshalb ist es wichtig, die gestiegenen und weiter steigenden Gehälter bei den Personalkosten einzuplanen. Betriebswirtschaftlich gesehen lassen sich stei- gende Gehälter bei den Angestellten in einigen Praxen jedoch nur bis zu einem bestimmten Punkt realisieren, ohne dass eine Anpassung bei den zahnärztlichen Leistungen stattfindet. Dass dies jedoch politisch - sowohl bei den gesetzlichen als auch bei den privaten Krankenversicherungen - nicht umgesetzt wird, zeigte zuletzt 2012 die Reformierung der Gebührenordnung für Zahnärzte (GOZ).

Neugründer sollten diese Entwicklung im Auge behalten, um realistische laufende Kosten zu ermitteln. Abgesehen von dem rein wirtschaftlichen Aspekt gibt es eine steigende Anzahl an neuapprobierten Zahnärzten bei weiterhin zu wenigen ZFA. Hier werden sich Kollegen in den nächsten Jahren mehr und mehr mit der Situation konfrontiert sehen, ohne eine ausreichende Versorgung durch ZFA arbeiten zu müssen.

In unseren unmittelbaren Nachbarländern, wie zum Beispiel Frankreich, gehört die Behandlung ohne Helferin zum Standard und wird schon während des Studiums dementsprechend vermittelt. Hierzulande darf man sich bisher noch auf eine Assistenz verlassen und wird auch dementsprechend ausgebildet.

Demographisch gesehen sind Regionen mit einer hohen Lebensqualität wie beispielsweise Ballungszentren auch von einer höheren Arbeitnehmerfluktuation betroffen. Hier werden sich frisch approbierte Kollegen in Zukunft vermehrt auf das „Einzelbehandlersystem“ einstellen müssen.

\section{Impressum}

Eigentümer \& Copyright @ Springer-Verlag Berlin Heidelberg 2015, Springer Medizin c/o Springer-Verlag GmbH, Tiergartenstr. 17, 69121 Heidelberg, Tel. +49 6221/487-0 Springer Medizin ist ein Teil der Fachverlagsgruppe Springer Science+Business Media Geschäftsführung Springer Medizin:

Joachim Krieger (President Professional Businesses), Fabian Kaufmann

Director Apotheke und Zahnmedizin: Marion Bornemann

Redaktionsleitung "der junge zahnarzt":

Dr. Swanett Koops (Tel. -8217, swanett.koops@springer.com)

Redaktion CME-Rubrik: Dr. Swanett Koops (Tel. -8217, swanett.koops@springer.com)

Layout: Arnulf Illing (Tel. -8952, arnulf.illing@springer.com)

Bildredaktion: Christiane Seufert

Anzeigenleitung: Marita Säuberlich (Tel. -8309, Fax -68309,

marita.saeuberlich@springer.com)

Corporate Publishing: Anja Weisse (Tel. -8739, Fax-68739, anja.weisse@springer.com)

Druck: Stürtz GmbH, Würzburg. Printed in Germany

Erscheinungsweise: 4 Ausgaben pro Jahr

Papierausgabe: ISSN 1869-5744, gedruckt auf säurefreiem Papier

Bezugspreise (unverb. Preisempfehlung inkl. 7\% deutscher MwSt. und Versand): Vorzugspreis für persönliche Abonnenten: EUR 56,-; Einzelheftpreis: EUR 22,--. Das Abonnement kann jederzeit 2 Monate vor Ende des Bezugszeitraumes gekündigt werden. Bestellungen oder Rückfragen nimmt jede Buchhandlung oder der Verlag entgegen.
Kontakt: Springer Customer Service Center GmbH, Haberstr. 7, 69126 Heidelberg, Tel. +49 6221/345-4303, Fax-4229, Leserservice@springer.com (Mo.-Fr. 8.00 Uhr bis 20.00 Uhr) Copyright \& allgemeine Hinweise: Die Zeitschrift sowie alle in ihr enthaltenen einzelnen Beiträge und Abbildungen sind urheberrechtlich geschützt. Jede Verwertung, die nicht ausdrücklich vom Urheberrechtsgesetz zugelassen ist, bedarf der vorherigen schriftlichen Zustimmung des Verlags. Das gilt insbesondere für Vervielfältigungen, Bearbeitungen, Übersetzungen, Mikroverfilmungen und die Einspeicherung und Verarbeitung in elektronischen Systemen.

Gezeichnete Artikel geben nicht unbedingt die Meinung der Redaktion wieder. Autoren können unter bestimmten Voraussetzungen an der Ausschüttung der Bibliotheks- und Fotokopietantiemen teilnehmen. Einzelheiten bei VG WORT, Abt. Wissenschaft, Goethestr. 49, 80336 München.

Angaben über Dosierungsanweisungen und Applikationsformen sind anhand anderer Literaturstellen oder der Packungsbeilage auf ihre Richtigkeit zu überprüfen. Der Verlag übernimmt keine Gewähr.

Der IVW (Informationsgemeinschaft zur Feststellung der Verbreitung von Werbeträgern) angeschlossen. 